\title{
Accepting stem cells
}

The rise of the embryonic stem cell has been swift. Fuelled by President George W. Bush's debate on whether to limit federal funding for human embryonic stem cell research, stem cells became a political cause $d u$ jour. Scientists, patient advocates and other proponents of stem cell research described utopian benefits from stem cells to cure debilitating diseases or grow replacement tissues. Opponents of embryonic stem cell research pointed out the moral costs of using these cells, the debate morphing into another platform for some anti-abortion activists. However, as ethical and legal issues became the central focus of this debate, certain important scientific facts were overlooked. One issue seemingly forgotten by both sides is only too familiar to immunologists: the problem of transplant rejection of allogeneic embryonic stem cells or tissues derived from them.

The immune system has evolved to seek out and destroy pathogens. As such, transplantation of tissues from genetically nonidentical individuals activates an immune response that rejects the transplanted tissue. Currently the only way to prevent rejection is to use immunosuppressive drugs. However, these are far from ideal. The side-effects of long-term use include opportunistic infections, drug-related toxicities, cancer and diabetes. The establishment of the Immune Tolerance Network in 1999 by the NIAID should facilitate the clinical application of immune tolerance-inducing strategies. But at present, the rejection process remains a hurdle. With this in mind, will transplantation of allogeneic embryonic stem cells be subject to the same obstacles as solid organ transplantation?

It has been suggested that embryonic stem cells may provoke less of an immune response than solid organ transplants. However, this may not be true of the differentiated tissue derived from the embryonic stem cells. Major histocompatibility complex (MHC) antigen expression, and therefore immunogenicity, will depend upon the cell type into which the stem cells differentiate. Immunogenicity is increased in the presence of inflammation or of signals that up-regulate costimulatory molecules and attract antigen-presenting cells (APCs). Tissue derived in vitro from embryonic stem cells would lack APCs and could, theoretically, induce a weaker immune response (the removal of these cells from tissues increases graft survival in some cases), but the transplantation procedure itself could induce inflammation. Unfortunately, no solid body of work has addressed these issues, but most immunologists would agree that rejection issues need resolution before stem cell technology can succeed.
A number of alternatives to immunosuppressants have been suggested that could encourage acceptance of tissue grafts derived from embryonic stem cells. The creation of a large pool of embryonic stem cell lines would increase the chances of matching MHC antigens. However, more than a million different lines would be required to create a stem cell bank, a far cry from the 72 embryonic stem cells lines that the US government has decided to further support. However, rejection would most likely still occur in the absence of immunosuppressive drugs: a result of minor histoincompatibilities. An alternative is to make embryonic stem cells less immunogenic by eliminating or introducing surface antigens through genetic engineering. But even if this were possible, the potential of these lines to accumulate genetic damage over time is high. Or perhaps transplant rejection could be circumvented by transferring recipient somatic cell nuclei into existing embryonic stem cell lines. Tissues would then be derived essentially from autologous stem cells, thus avoiding allogeneic incompatibilities. Nevertheless, this procedure is highly controversial, as it uses techniques developed for reproductive cloning. In terms of both rejection and ethics, autologous adult stem cells taken from the graft recipient could be the answer, but it is unclear at present how multipotential these cells really are. Genetic modification of these cells would be required for treatment of many congenital disorders. In addition, adult stem cells are rare, difficult to identify and purify and, when grown in culture, are hard to maintain in an undifferentiated state.

Clearly, stem cell research is in its infancy. The rejection issue is just as real as the moral problems. Unfortunately, it is only now-after policy decisions have been made - that scientists have begun to publicly point out the practical problems, such as genetic instability and insufficient diversity, inherent in relying on a limited number of cell lines. Overcoming the rejection of transplanted stem cells will require much research and more federal funding. The moral concerns over the use of human stem cells pushed other issues - issues that should have been prominent in the public discussion - to the sidelines. Did proponents avoid discussing transplant rejection because it may have played into the hands of the opposition? The failure to be open has given the general public, whether they agree with their use or not, the impression that stem cells will "cure all evils". Whatever the reasons for not debating the issue, immunological rejection of stem cell-derived tissues is a problem that needs research, funding and resolution. 